\title{
Workflow and factors associated with delay in the delivery of intra-arterial treatment for acute ischemic stroke in the MR CLEAN trial
}

\author{
Esmee Venema, ${ }^{1,2}$ Nikki Boodt, ${ }^{2}$ Olvert A Berkhemer, ${ }^{3,4,5}$ Pleunie P M Rood, ${ }^{6}$ \\ Wim H van Zwam, ${ }^{4}$ Robert J van Oostenbrugge, ${ }^{7}$ Aad van der Lugt, ${ }^{3}$ \\ Yvo B W E M Roos, ${ }^{8}$ Charles B L M Majoie, ${ }^{5}$ Hester F Lingsma, ${ }^{1}$ Diederik W J Dippel, ${ }^{2}$ \\ on behalf of the MR CLEAN investigators
}

\begin{abstract}
- Additional material is published online only. To view please visit the journal online (http://dx.doi.org/10.1136/ neurintsurg-2017-013198).
\end{abstract}

For numbered affiliations see end of article.

\section{Correspondence to}

Esmee Venema, Department of Public Health and Department of Neurology, Erasmus University Medical Center, P.O. Box 2040, 3000 CA Rotterdam, The Netherlands; e.venema@ erasmusmc.n

EV and NB contributed equally.

Received 12 May 2017 Revised 23 July 2017 Accepted 30 July 2017 Published Online First 30 August 2017
Check for updates

To cite: Venema $\mathrm{E}$, Boodt $\mathrm{N}$, Berkhemer OA, et al. J Neurolntervent Surg 2018:10:424-428

\section{ABSTRACT}

Objective The effect of intra-arterial treatment (IAT) for acute ischemic stroke is highly time-dependent. We investigated the delay of IAT and factors associated with such delay.

Methods MR CLEAN was a randomized trial of IAT plus usual care versus usual care alone $(n=500)$. With multivariable linear regression, we analyzed the effect of intravenous treatment, general anesthesia, off-hours and inter-hospital transfer on time to admission to the emergency department (ED) of the intervention center and time to treatment. Furthermore, we assessed compliance with a target of $75 \mathrm{~min}$ for time from ED to treatment, and calculated the potential absolute increase in the number of patients with a good outcome (modified Rankin Scale score $\leq 2$ ) if this target had been achieved in all treated patients.

Results Inter-hospital transfer prolonged time to ED by $140 \mathrm{~min}(95 \% \mathrm{Cl} 129$ to 150$)$ but reduced time from ED to treatment by $77 \mathrm{~min}(95 \% \mathrm{Cl} 64$ to 91$)$. Time from ED to treatment was increased by 19 min by general anesthesia ( $95 \% \mathrm{Cl} 5$ to 33 ) and total time was increased by 23 min during off-hours ( $95 \% \mathrm{Cl} 6$ to 40 ). The in-hospital target was achieved in 11.5\% (22/192) of patients. Full compliance with the target time of 75 min from ED to treatment would have increased the proportion of patients with a good outcome by $7.6 \%$ (95\% Cl 6.7\% to $8.5 \%$ ).

Conclusion Inter-hospital transfer is an important cause of delay in the delivery of IAT and every effort should be made to avoid transfers and reduce transferrelated delay. Furthermore, in-hospital workflow should be optimized to improve functional outcome after IAT.

\section{INTRODUCTION}

Intra-arterial treatment (IAT) with the use of stent retrievers has been proved to be safe and effective for patients with acute ischemic stroke due to a proximal intracranial arterial occlusion in the anterior circulation. ${ }^{1}$ The effect of treatment is highly time-dependent and increased time from stroke onset to reperfusion is associated with a decreased likelihood of good functional outcome. ${ }^{2-7}$ The Multicenter Randomized Clinical Trial of Endovascular Treatment for Acute Ischemic Stroke in the Netherlands (MR CLEAN) showed a decrease in the effect of IAT on good outcome (risk difference between intervention and control) of $6.4 \%$ per hour delay in time to expected reperfusion. ${ }^{3}$ It seems likely that reducing treatment delay will further increase the effect of IAT.

The timeline from stroke onset to reperfusion consists of several steps, and each step is prone to factors causing delay. An important potential cause of delay in the prehospital stage is referral to a community hospital without facilities for IAT (a primary stroke center), which necessitates inter-hospital transportation of patients to an intervention center. Other factors that may increase time to reperfusion are treatment with intravenous tissue plasminogen activator (IV tPA), the use of general anesthesia during the endovascular procedure, and inefficiency of in-hospital workflow, specifically outside working hours. ${ }^{8-10}$ In-hospital workflow consists of all protocols and procedures in the emergency department (ED) of the primary stroke center, the ED of the intervention center, and the interventional suite.

In addition to the previously defined benchmark time of $60 \mathrm{~min}$ for treatment with IV tPA, various lenient or strict target times for IAT are now being suggested to improve in-hospital workflow. ${ }^{11-13}$ The Highly Effective Reperfusion Evaluated in Multiple Endovascular Stroke Trials (HERMES) collaborators reported the $25^{\text {th }}$ percentile of ED admission to groin puncture, which amounted to $75 \mathrm{~min}$, and could very well serve as a benchmark time. ${ }^{7}$

Our aim was to investigate prehospital and in-hospital delay in the delivery of IAT in the MR CLEAN trial and to assess the impact of inter-hospital transfer and other factors on such delay.

\section{METHODS}

\section{Study design}

MR CLEAN was a multicenter randomized clinical trial in the Netherlands comparing IAT plus usual care (intervention group) with usual care alone (control group). MR CLEAN is registered with the ISRCTN registry ISRCTN10888758. ${ }^{14} 15$

\section{Study setting and population}

Patients aged 18 years or older with a clinical diagnosis of acute stroke, a deficit on the National 
Institute of Health Severity of Stroke (NIHSS) scale of 2 points or more, and a proximal occlusion in the anterior circulation on vessel imaging were included. Detailed inclusion and exclusion criteria can be found in the study protocol. ${ }^{14}$ Usual care could include treatment with IV tPA if indicated. The endovascular procedure had to be initiated within 6 hours after onset of stroke symptoms and could include intra-arterial delivery of a thrombolytic agent at the level of occlusion, mechanical thrombectomy, or both. In total, 500 patients were enrolled from December 2010 to March 2014. Of these patients, 233 were allocated to the intervention arm and 267 were allocated to the control arm. IAT consisted of stent thrombectomy in the majority of cases $(195 / 233,84 \%) .{ }^{14} 15$

\section{Study protocol}

Inclusion in the study could occur in three settings: (1) patients arriving directly at an intervention center after onset of stroke symptoms; (2) patients presenting at a primary stroke center first before being transferred to an intervention center for study participation; or (3) patients suffering a stroke while already hospitalized. In the hospital of first presentation, a non-contrast CT (NCCT) scan was performed to exclude intracerebral hemorrhage. This was followed by standard medical treatment which could include IV tPA. Vessel imaging with computed tomographic angiography (CTA), magnetic resonance angiography (MRA), or digital subtraction angiography (DSA) had to be performed before inclusion in the study in the intervention center to assess the presence of an intracranial arterial occlusion.

Time points of various events were prospectively collected in the trial, including time of stroke onset, start of treatment with IV t-PA, admission to the ED of the intervention center, and time of randomization. In patients receiving IAT, timing of treatment was documented by the local interventionist. Time of imaging (ie, NCCT and CTA, MRA or DSA) was prospectively recorded in the intervention centers, but had to be collected retrospectively if performed in a primary stroke center.

\section{Definitions and outcome measures}

Stroke onset was defined as the time point when stroke sympcases where the time of first symptoms was unknown, stroke onset was defined as the moment the patient was last seen well. We defined off-hours as weekend days or working days between 17:00 hours and 08:00 hours. Groin puncture was defined as the moment of placing a sheet in the groin, indicating the start of the endovascular procedure. Reperfusion was measured with the modified Thrombolysis in Cerebral Infarction (mTICI) score, which can range from grade 0 for no reperfusion to grade 3 for full reperfusion. ${ }^{16}$ We defined time of reperfusion as the first moment a mTICI score of $2 \mathrm{~b}$ or 3 was reached. When reperfusion was not achieved we used the timepoint when the last angiography run was done, which indicated the end of the endovascular procedure.

Time intervals of primary interest were: (1) time from stroke onset to admission to the ED of the intervention center as a measure for prehospital delay; (2) time from admission to the $\mathrm{ED}$ of the intervention center to groin puncture as a measure for in-hospital delay; and (3) time from stroke onset to groin puncture. Additionally, we compared the following time intervals between transfer patients and direct patients: (4) time from stroke onset to treatment with IV tPA; (5) time from admission to ED to vessel imaging; (6) time from vessel imaging to randomization; and (7) time from groin puncture to reperfusion. If all vessel imaging was performed in the primary stroke center, the toms were first noticed by the patient or an observer. In

patient was excluded from analyses (5) and (6). Time of transportation from the primary stroke center to the intervention center was estimated for all transfer patients with the TomTom MyDrive application (Version 4.1.4.3089, available on https:// mydrive.tomtom.com).

\section{Statistical analysis}

For descriptive purposes, time intervals were reported as medians with IQR. To assess transfer-related treatment delay we compared the baseline characteristics and median time intervals of patients transferred from a primary stroke center with those arriving directly at the intervention center.

Using multivariable linear regression analyses we evaluated the effect of patient and workflow-related characteristics on time to treatment. Factors assessed were administration of IV $\mathrm{t}$-PA, use of general anesthesia during the endovascular procedure, admission during off-hours, and inter-hospital transfer. We included the following potential confounders in these analyses: age, gender, baseline NIHSS score, history of ischemic stroke, and prestroke modified Rankin Scale (mRS). Missing baseline characteristics were imputed with the mean (continuous variables) or mode (categorical variables). Missing time variables were not imputed.

To assess the impact of in-hospital delay, we compared the time from admission to the ED to groin puncture with the target time of $75 \mathrm{~min}$. For each individual patient we estimated the excess delay by subtracting the target time from the actual time. The mean excess delay of all treated patients was multiplied by an increase in treatment benefit (defined as the risk difference for good outcome (mRS score $\leq 2)$ ) of $6.4 \%$ per hour, based on previous results of the MR CLEAN trial. ${ }^{3}$ This provides an estimate of the potential increase in treatment benefit if the target time had been achieved in all treated patients.

All statistical analyses were performed with $\mathrm{R}$ software (Version 3.2.1, R Foundation).

\section{RESULTS}

Of the 500 patients included in the MR CLEAN trial, 281 $(56.2 \%)$ arrived directly at an intervention center and 219 $(43.8 \%)$ were transferred from a primary stroke center. Transfer patients were significantly younger than those arriving directly at an intervention center (62.2 vs 67.0 years) and were admitted more frequently during off-hours ( $63.4 \%$ vs $51.6 \%)$. Other baseline characteristics are shown in table 1.

Median time from stroke onset to groin puncture was $233 \mathrm{~min}$ (IQR 202-287) in direct arrival patients and $315 \mathrm{~min}$ (IQR 260-346) in transfer patients (table 2).

The median time of inter-hospital transportation was $30 \mathrm{~min}$ (IQR 23-41), but total time from onset to admission to the $\mathrm{ED}$ of the intervention center was 148 min longer in transfer patients than in direct arrival patients. In-hospital workflow was improved in transfer patients, with a decrease in median time from arrival to vessel imaging of $15 \mathrm{~min}$ and a decrease in time from vessel imaging to randomization of $32 \mathrm{~min}$. Duration of the endovascular procedure from groin puncture to reperfusion was comparable $(56 \mathrm{~min}$ in direct arrival patients vs $61 \mathrm{~min}$ in transfer patients). Overall, we observed an association between increasing time from onset of stroke to admission to ED and decreasing time from admission to groin puncture (online supplementary material figure 1 ). However, the average prehospital delay caused by inter-hospital transfer was only partially compensated by improved in-hospital workflow (figure 1).

In multivariable analysis, the total time to treatment (groin puncture) was increased by 23 min during off-hours (95\% CI 6 
Table 1 Baseline characteristics of patients in the MR CLEAN trial according to mode of arrival at the emergency department of the intervention center

\begin{tabular}{|c|c|c|c|}
\hline & $\begin{array}{l}\text { Direct } \\
(n=281)\end{array}$ & $\begin{array}{l}\text { Transfer } \\
(\mathrm{n}=219)\end{array}$ & $\mathrm{p}$ Value \\
\hline Age, years, mean (SD) & $67.0(13.6)$ & $62.2(13.6)$ & $<0.01$ \\
\hline Male sex, n (\%) & $156(55.5)$ & $136(62.1)$ & 0.16 \\
\hline \multicolumn{4}{|l|}{ Medical history, n (\%) } \\
\hline Atrial fibrillation & $77(27.4)$ & $58(26.5)$ & 0.90 \\
\hline Hypertension & $135(48.0)$ & $92(42.0)$ & 0.21 \\
\hline Hyperlipidemia & $71(25.3)$ & $58(26.5)$ & 0.84 \\
\hline Diabetes mellitus & $41(14.6)$ & $27(12.3)$ & 0.55 \\
\hline Previous ischemic stroke & $33(11.7)$ & $21(9.6)$ & 0.53 \\
\hline Prestroke mRS, n (\%) & & & 0.09 \\
\hline 0 & $220(78.3)$ & $184(84.0)$ & \\
\hline 1 & $27(9.6)$ & $23(10.5)$ & \\
\hline 2 & $19(6.8)$ & $6(2.7)$ & \\
\hline$>2$ & $15(5.3)$ & $6(2.7)$ & \\
\hline NIHSS score, median (IQR) & $\begin{array}{l}17(14 \text { to } \\
22)\end{array}$ & 18 (15 to 22$)$ & 0.13 \\
\hline Location of occlusion, $n(\%)^{*}$ & & & 0.24 \\
\hline Internal carotid artery & $72(25.7)$ & $66(30.1)$ & \\
\hline M1 middle cerebral artery segment & $180(64.3)$ & $139(63.5)$ & \\
\hline M2 middle cerebral artery segment & $27(9.6)$ & $12(5.5)$ & \\
\hline $\begin{array}{l}\text { A1 or } A 2 \text { anterior cerebral artery } \\
\text { segment }\end{array}$ & $1(0.4)$ & $2(0.9)$ & \\
\hline Treatment with IV tPA, n (\%) & $249(88.6)$ & $196(89.5)$ & 0.87 \\
\hline Admission during off-hours, $\mathrm{n}(\%) \dagger$ & $141(51.6)$ & $128(63.4)$ & 0.01 \\
\hline Allocation to IAT, n (\%) & $132(47.0)$ & $101(46.1)$ & 0.92 \\
\hline Use of general anesthesia, $n(\%) \ddagger$ & 39 (32.5) & $41(42.3)$ & 0.18 \\
\hline $\begin{array}{l}\text { *0ne missing value. } \\
\text { †25 missing values. } \\
\text { ‡Only intra-arterial treated patients. } \\
\text { IAT, intra-arterial treatment; IV tPA, intr } \\
\text { mRS, modified Rankin Scale; NIHSS, Nat }\end{array}$ & Institutes of & terce & \\
\hline
\end{tabular}

Table 2 Time intervals (min) in the MR CLEAN trial according to mode of arrival at the emergency department of the intervention center, expressed as median (IQR)

\begin{tabular}{|c|c|c|}
\hline All patients & $\begin{array}{l}\text { Direct } \\
(n=281)\end{array}$ & $\begin{array}{l}\text { Transfer } \\
(n=219)\end{array}$ \\
\hline Stroke onset to IV tPA $(n=444)$ & $85(65-110)$ & $85(66-110)$ \\
\hline Stroke onset to admission to $E D(n=471)$ & $52(34-75)$ & $200(166-245)$ \\
\hline $\begin{array}{l}\text { Admission to ED to vessel imaging } \\
(n=439)\end{array}$ & $39(25-67)$ & $24(17-32)$ \\
\hline Vessel imaging to randomization $(n=460)$ & $53(34-80)$ & $21(15-33)$ \\
\hline Intra-arterial treated patients & $\begin{array}{l}\text { Direct } \\
(n=132)\end{array}$ & $\begin{array}{l}\text { Transfer } \\
(n=101)\end{array}$ \\
\hline Stroke onset to groin puncture $(n=205)$ & $233(202-287)$ & $315(260-346)$ \\
\hline $\begin{array}{l}\text { Admission to ED to groin puncture } \\
(n=192)\end{array}$ & $170(142-205)$ & $96(75-120)$ \\
\hline Groin puncture to reperfusion $(n=194)$ & $56(42-86)$ & $61(37-90)$ \\
\hline
\end{tabular}

D, emergency department; IV tPA, intravenous tissue plasminogen activator. to 40 ) and by 65 min due to inter-hospital transfer (95\% CI 48 to 82 ) (figure 2). Transfer prolonged time to admission to the ED by $140 \mathrm{~min}(95 \% \mathrm{CI} 129$ to 150$)$, but was associated with a shorter time to groin puncture $(77 \mathrm{~min}, 95 \% \mathrm{CI} 64$ to 91 ). Time from ED to groin puncture was increased by an additional 19 min by the use of general anesthesia during the endovascular procedure (95\% CI 5 to 33 ).

In patients receiving IAT, time from admission to the ED to groin puncture was on average $145 \pm 61 \mathrm{~min}$. The defined target time of $75 \mathrm{~min}$ was reached in only $11.5 \%$ (22/192) of the treated patients. However, if this target time had been achieved in all patients, mean time from onset to groin puncture would have been $71 \mathrm{~min}$ (95\% CI 63 to 80 ) shorter. Because the risk difference of good functional outcome decreases by $6.4 \%$ per hour, the absolute treatment benefit (proportion of patients with mRS 0-2) in MR CLEAN could have been 7.6\% (95\% CI 6.7\% to $8.5 \%$ ) higher.

\section{DISCUSSION}

This study shows that inter-hospital transfer was the most important factor associated with treatment delay in the MR CLEAN trial. Time from onset to treatment increases by more than 1 hour in patients transferred from a primary stroke center, despite improved in-hospital workflow times. Furthermore, only a few patients received IAT within the proposed target time of $75 \mathrm{~min}$ for time from admission to the ED to groin puncture, and enhancing this compliance by improving in-hospital workflow might further increase functional outcome after IAT.

Overall, the time intervals in MR CLEAN are longer than in several other trials of IAT such as the Endovascular Treatment for Small Core and Proximal Occlusion Ischemic Stroke (ESCAPE), EXtending the time for Thrombolysis in Emergency Neurological Deficits with Intra-Arterial therapy (EXTEND-IA), and Solitaire With the Intention for Thrombectomy as Primary Endovascular Treatment (SWIFT PRIME). ${ }^{17-19}$ For example, median time to reperfusion was $340 \mathrm{~min}$ in MR CLEAN versus $241 \mathrm{~min}$ in ESCAPE. This can be explained by the strong focus on improving workflow and reducing time delays in ESCAPE, while MR CLEAN did not require the participating investigators to be trained in delivery of fast care and logistics. Furthermore, the study design of these trials allowed verbal or deferred informed consent while, in MR CLEAN, written informed consent was required before randomization. ${ }^{15} 18$ Trials with a shorter median time to groin puncture had a significantly larger treatment effect (figure 2 in online supplementary material).

Total time to treatment was increased most by inter-hospital transfer, confirming the results of previous studies. ${ }^{45} 910$ Transfer patients arrived $148 \mathrm{~min}$ later at the ED, while the median transportation time in our study was only $30 \mathrm{~min}$. This suggests that most delay was caused during work-up in the primary hospital. However, increased time to arrival at the ED was clearly associated with a decrease in the following time to groin puncture. This paradoxical effect was likely caused by the fact that this group consisted mainly of transfer patients who had already undergone part of the work-up, the intervention center was pre-notified and clinical information was available before arrival at the ED, which made it possible for the staff to mobilize the neurointerventional team and for the interventional suite to be prepared in advance. This finding suggests that improvement of in-hospital workflow might lead to a shorter time from ED to treatment for patients who are directly admitted to an intervention center. 


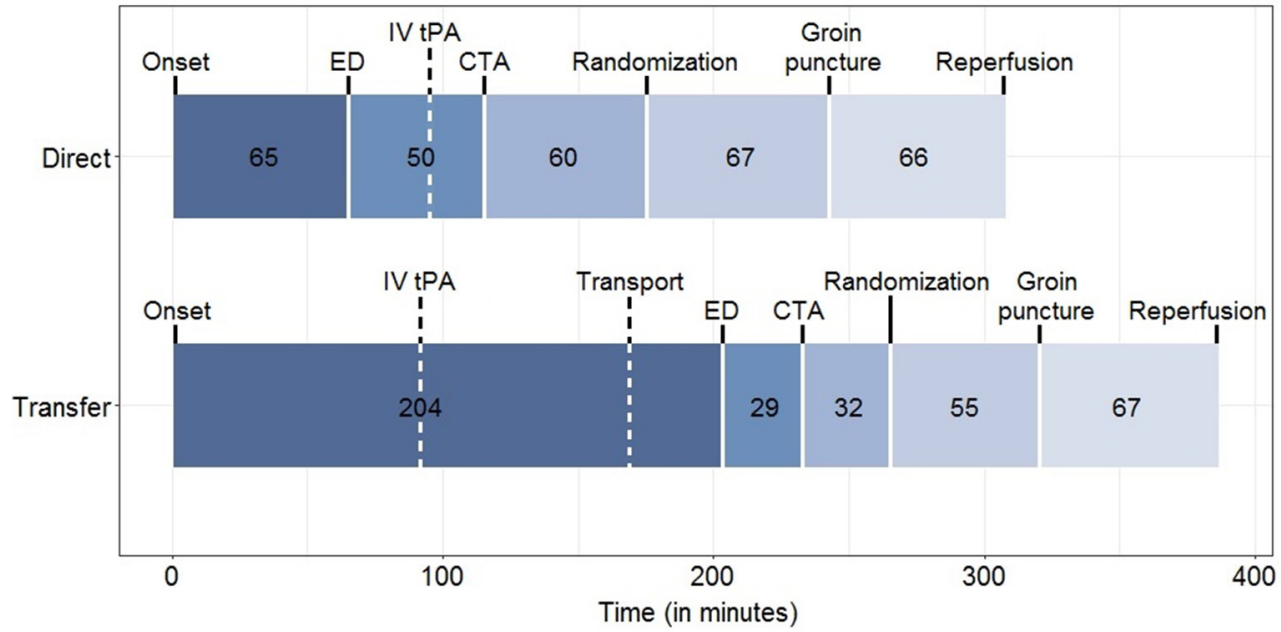

Figure 1 Mean time intervals in the MR CLEAN trial $(n=500)$ according to mode of arrival at the emergency department of the intervention center. CTA, computed tomography angiography; ED, emergency department; IV tPA, intravenous tissue plasminogen activator.

Our study has a number of limitations. Our analysis was restricted to data that were collected during the trial. We had no information available on the time of arrival in the primary stroke center and the time of departure to the intervention center to estimate door-in to door-out times, which might have provided more insight into the various elements of delay. Also, the reported workflow times from this randomized study might not be representative of daily clinical practice, since the process of obtaining written informed consent and randomization can increase treatment delay substantially. Furthermore, vessel imaging in the MR CLEAN trial was often performed or repeated in the intervention center while, in daily routine, transfer patients can be directly transferred to the interventional suite without any delay of additional imaging. Since the distances between hospitals in the Netherlands are very short and the median transportation time in this study was only $30 \mathrm{~min}$, our results cannot be easily extrapolated to other countries. Transportation times in larger countries are usually longer, and the effect of inter-hospital transfer on treatment delay might therefore be much greater in those countries. Last, inter-hospital transfer has a disadvantageous effect on time to treatment and also on the number of patients who might be eligible for treatment. ${ }^{20}$ We had no data on the patients in primary stroke centers who were not included in the trial because of the need for a transfer and the associated delay. Therefore we were not able to quantify the impact of transfer delay on the likelihood of receiving IAT.

Overall, it remains clear that inter-hospital transfer causes significant prehospital delays in IAT, and that adequate

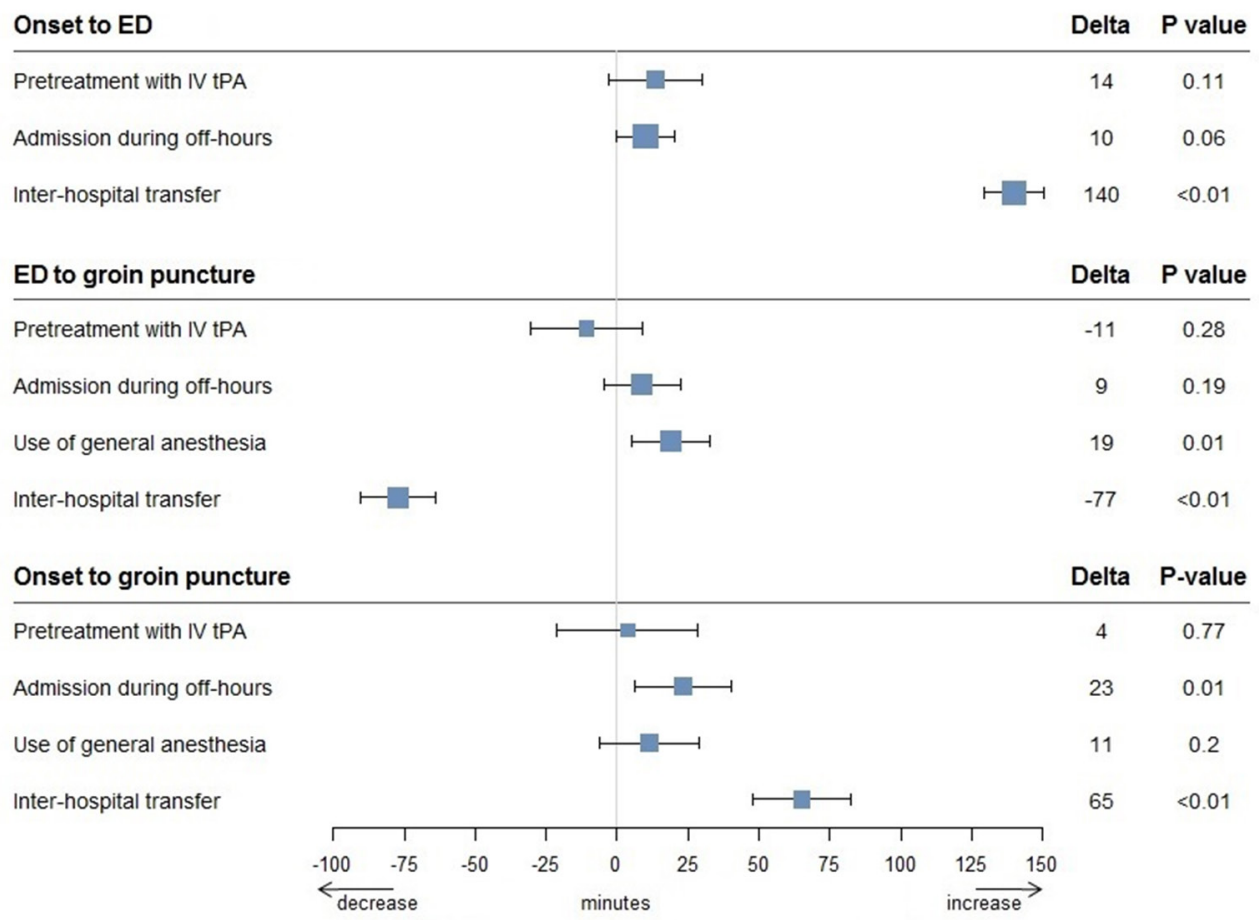

Figure 2 Effect of clinical factors on time intervals in the MR CLEAN trial $(n=500)$. ED, emergency department; IV tPA, intravenous tissue plasminogen activator. 
measures have to be taken to shorten transfer times and, if possible, to reduce the number of transferred patients. This could be achieved by transporting patients with acute ischemic stroke directly to an intervention center using prehospital triaging scales to select patients with a high risk of a large vessel occlusion. ${ }^{21-23}$ These scales have to be implemented in regional care systems and protocols for prehospital triage to minimize transfer delays depending on the characteristics of the area. ${ }^{24-26}$ In regions with very large distances, the additional use of air ambulance services for the transportation of IAT candidates might be beneficial. Another possibility would be to bypass the primary stroke center by using a mobile stroke unit; an ambulance with a CT scanner and a specialized team on board, allowing in-ambulance initiation and administration of IV tPA and direct transportation to the intervention center. $^{27-29}$

The other important step in reducing treatment delay in IAT is improving in-hospital workflow. The reduced time from ED to groin puncture in transfer patients suggests that it should be possible to shorten the time delay in the ED. Previous studies have already shown that optimizing workflow and protocols can decrease time to treatment. ${ }^{31}$ Pre-notification of the interventional team as soon as a patient with a suspected large vessel occlusion is announced, direct transport from door to scanner, a parallel nature of care processes and optimizing the processes around IV tPA administration and initiation of IAT are all potential factors to reduce ED delay. ${ }^{10}{ }^{32} 33$ Providing reasonable time targets might also improve this process, as was shown with the introduction of door-to-balloon target times for percutaneous coronary intervention. ${ }^{34}$

In summary, inter-hospital transfer is an important cause of delay in the delivery of IAT and every effort should be made to avoid transfer and reduce transfer-related delay. Furthermore, in-hospital workflow can and should be optimized to improve functional outcome after IAT.

\author{
Author affiliations \\ 'Department of Public Health, Erasmus University Medical Center, Rotterdam, The \\ Netherlands \\ ${ }^{2}$ Department of Neurology, Erasmus University Medical Center, Rotterdam, The \\ Netherlands \\ ${ }^{3}$ Department of Radiology, Erasmus University Medical Center, Rotterdam, The \\ Netherlands \\ ${ }^{4}$ Department of Radiology, Maastricht University Medical Center, Maastricht, The \\ Netherlands \\ ${ }^{5}$ Department of Radiology, Academic Medical Center, Amsterdam, The Netherlands \\ ${ }^{6}$ Department of Emergency Medicine, Erasmus University Medical Center, Rotterdam, \\ The Netherlands \\ ${ }^{7}$ Department of Neurology, Maastricht University Medical Center, Maastricht, The \\ Netherlands \\ ${ }^{8}$ Department of Neurology, Academic Medical Center, Amsterdam, The Netherlands
}

Contributors WHZ, RJvO, AvdL, YBWEMR, CBLMM and DWJD conceived the study, designed the trial, and obtained research funding. OAB supervised the conduct of the trial and data collection. EV and NB analyzed the data and drafted the manuscript (authors contributed equally). HFL provided statistical advice. All authors contributed substantially to its revision. DWJD takes responsibility for the paper as a whole.

Funding The Multicenter Randomized Clinical Trial of Endovascular Therapy for Acute Ischemic Stroke in the Netherlands (MR CLEAN) was partly funded by the Dutch Heart Foundation and by unrestricted grants from AngioCare BV, Medtronicl Covidien/EV3, MEDAC Gmbh/LAMEPRO, Penumbra, Stryker, and Top Medical/ Concentric.

Competing interests Academic Medical Center received funds from Stryker for consultations by YBWEMR and CBLMM. Erasmus University Medical Center received funds from Stryker for consultations by DWJD and AvdL, and from Bracco Imaging for consultations by DWJD. Maastricht University Medical Center received funds from Codman and Stryker for consultations by WHZ. The other authors report no conflicts.

Ethics approval The trial protocol was approved by all ethical boards of the participating centers, and all participants (or their legal representatives) provided written informed consent.

Provenance and peer review Not commissioned; externally peer reviewed.

Data sharing statement No additional data available.

(C) Article author(s) (or their employer(s) unless otherwise stated in the text of the article) 2018. All rights reserved. No commercial use is permitted unless otherwise expressly granted.

\section{REFERENCES}

1 Goyal M, Menon BK, van Zwam WH, et al. Endovascular thrombectomy after large-vessel ischaemic stroke: a meta-analysis of individual patient data from five randomised trials. Lancet 2016;387:1723-31.

2 Khatri P, Yeatts SD, Mazighi M, et al. Time to angiographic reperfusion and clinical outcome after acute ischaemic stroke: an analysis of data from the Interventional Management of Stroke (IMS III) phase 3 trial. Lancet Neurol 2014;13:567-74

3 Fransen PS, Berkhemer OA, Lingsma HF, et al. Time to reperfusion and treatment effect for acute ischemic stroke: a randomized clinical trial. JAMA Neurol 2016;73:1-7.

4 Goyal M, Jadhav AP, Bonafe A, et al. Analysis of workflow and time to treatment and the effects on outcome in endovascular treatment of acute ischemic stroke: results from the SWIFT PRIME randomized controlled trial. Radiology 2016;279:888-97.

5 Menon BK, Sajobi TT, Zhang Y, et al. Analysis of workflow and time to treatment on thrombectomy outcome in the endovascular treatment for small core and proximal occlusion ischemic stroke (ESCAPE) randomized, controlled trial. Circulation 2016:133:2279-86.

6 Ribo M, Molina CA, Cobo E, et al. Association between time to reperfusion and outcome is primarily driven by the time from imaging to reperfusion. Stroke 2016;47:999-1004.

7 Saver JL, Goyal M, van der Lugt A, et al. Time to treatment with endovascular thrombectomy and outcomes from ischemic stroke: a meta-analysis. JAMA 2016:316:1279-88.

8 Asif KS, Lazzaro MA, Zaidat O. Identifying delays to mechanical thrombectomy for acute stroke: onset to door and door to clot times. J Neurointerv Surg 2014:6:505-10.

9 Goyal M, Almekhlafi MA, Fan L, et al. Evaluation of interval times from onset to reperfusion in patients undergoing endovascular therapy in the Interventional Management of Stroke III trial. Circulation 2014;130:265-72.

10 Menon BK, Almekhlafi MA, Pereira VM, et al. Optimal workflow and processbased performance measures for endovascular therapy in acute ischemic stroke: analysis of the Solitaire FR thrombectomy for acute revascularization study. Stroke 2014:45:2024-9.

11 Casaubon LK, Boulanger JM, Blacquiere D, et al. Canadian stroke best practice recommendations: hyperacute stroke care guidelines, update 2015. Int J Stroke 2015:10:924-40.

12 Sacks D, Black CM, Cognard C, et al. Multisociety consensus quality improvement guidelines for intraarterial catheter-directed treatment of acute ischemic stroke, from the American Society of Neuroradiology, Canadian Interventional Radiology Association, Cardiovascular and Interventional Radiological Society of Europe, Society for Cardiovascular Angiography and Interventions, Society of Interventional Radiology, Society of Neurolnterventional Surgery, European Society of Minimally Invasive Neurological Therapy, and Society of Vascular and Interventional Neurology. AJNR Am J Neuroradiol 2013;34:E0.

13 McTaggart RA, Ansari SA, Goyal M, et al. Initial hospital management of patients with emergent large vessel occlusion (ELVO): report of the standards and guidelines committee of the Society of Neurolnterventional Surgery. J Neurointerv Surg 2017:9:316-23.

14 Fransen PS, Beumer D, Berkhemer OA, et al. MR CLEAN, a multicenter randomized clinical trial of endovascular treatment for acute ischemic stroke in the Netherlands: study protocol for a randomized controlled trial. Trials 2014;15:343.

15 Berkhemer OA, Fransen PS, Beumer D, et al. A randomized trial of intraarterial treatment for acute ischemic stroke. N Engl J Med 2015;372:11-20.

16 Zaidat 00 , Yoo AJ, Khatri $\mathrm{P}$, et al. Recommendations on angiographic revascularization grading standards for acute ischemic stroke: a consensus statement. Stroke 2013:44:2650-63.

17 Campbell BC, Mitchell PJ, Kleinig TJ, et al. Endovascular therapy for ischemic stroke with perfusion-imaging selection. N Eng/ J Med 2015;372:1009-18.

18 Goyal M, Demchuk AM, Menon BK, et al. Randomized assessment of rapid endovascular treatment of ischemic stroke. N Engl J Med 2015;372:1019-30.

19 Saver JL, Goyal M, Bonafe A, et al. Stent-retriever thrombectomy after intravenous t-PA vs. t-PA alone in stroke. N Engl J Med 2015;372:2285-95.

20 Prabhakaran $\mathrm{S}$, Ward $\mathrm{E}$, John $\mathrm{S}$, et al. Transfer delay is a major factor limiting the use of intra-arterial treatment in acute ischemic stroke. Stroke 2011;42:1626-30.

21 Pérez de la Ossa N, Carrera D, Gorchs M, et al. Design and validation of a prehospital stroke scale to predict large arterial occlusion: the rapid arterial occlusion evaluation scale. Stroke 2014;45:87-91. 
22 Lima FO, Silva GS, Furie KL, et al. Field assessment stroke triage for emergency destination: a simple and accurate prehospital scale to detect large vessel occlusion strokes. Stroke 2016;47:1997-2002.

23 Hastrup S, Damgaard D, Johnsen SP, et al. Prehospital acute stroke severity scale to predict large artery occlusion: design and comparison with other scales. Stroke 2016:47:1772-6.

24 Michel P. Prehospital scales for large vessel occlusion: closing in on a moving target. Stroke 2017:48:247-9.

25 Holodinsky JK, Williamson TS, Kamal N, et al. Drip and ship versus direct to comprehensive stroke center: conditional probability modeling. Stroke 2017;48:233-8.

26 Milne MS, Holodinsky JK, Hill MD, et al. Drip 'n ship versus mothership for endovascular treatment: modeling the best transportation options for optimal outcomes. Stroke 2017;48:791-4.

27 Ebinger $\mathrm{M}$, Winter $\mathrm{B}$, Wendt $\mathrm{M}$, et al. Effect of the use of ambulance-based thrombolysis on time to thrombolysis in acute ischemic stroke. JAMA 2014;311:1622-31.

28 Rajan SS, Rajan S, Baraniuk S, et al. Implementing a mobile stroke unit program in the United States: why, how, and how much? JAMA Neurol 2015;72:229-34.
29 Walter S, Kostopoulos P, Haass A, et al. Diagnosis and treatment of patients with stroke in a mobile stroke unit versus in hospital: a randomised controlled trial. Lancet Neurol 2012;11:397-404.

30 Aghaebrahim A, Streib C, Rangaraju S, et al. Streamlining door to recanalization processes in endovascular stroke therapy. J Neurointerv Surg 2017:9:340-5

31 Schregel K, Behme D, Tsogkas I, et al. Effects of workflow optimization in endovascularly treated stroke patients: a pre-post effectiveness study. PLoS One 2016:11:e0169192.

32 Zerna C, Assis Z, d'Esterre CD, et al. Imaging, intervention, and workflow in acute ischemic stroke: the Calgary approach. AJNR Am J Neuroradiol 2016;37:978-84.

33 Goyal M, Jadhav AP, Wilson AT, et al. Shifting bottlenecks in acute stroke treatment. J Neurointerv Surg 2016;8:1099-100.

34 Steg PG, James SK, Atar D, et al. ESC Guidelines for the management of acute myocardial infarction in patients presenting with ST-segment elevation. Eur Heart J 2012:33:2569-619. 